\title{
How to prevention and treatment the posthepatectomy liver failure: As a literature review from the latest research
}

\author{
Zulfa SAUMIA*
}

Department of History, Alumnus Universitas Gadjah Mada, Yogyakarta, Indonesia

Introduction: The liver has a unique ability to regenerate in a short period of time. This feature has driven the development of innovative strategies in liver surgery and transplantation. Liver anatomy is of utmost importance in considering advances in liver surgery. However, we sometimes have to deal with postepepatectomy liver failure (PHLF). PHLF is one of the most serious complications after liver resection and is still reported in up to $8 \%$ after liver resection. Post-hepatectomy liver failure as an impairment of the liver's ability to maintain synthetic, excretory, and detoxification functions, which is characterized by an increase in the international normalized ratio and concomitant hyperbilirubinemia. (according to local laboratory normal limits) on or after the postoperative day. The severity of PHLF should be assessed based on its impact on clinical management. What about the latest research on prevention and treatment in this PHLF?

Methods: The method used is to search for related articles using the keywords posthepatectomy, liver surgery etc. in PubMed and other medical journals.

Results: Although, surgical techniques and postoperative treatment have improved in recent years, PHLF treatment remains difficult. Lung, kidney, and circulatory disorders should be treated with a therapeutic regimen and it is necessary to make an early diagnosis of PHLF and start treatment in the intensive care unit to optimize and restore liver function.

Conclusions: It is necessary to make improvements in hospital and patient management to facilitate the prevention and treatment of posthepatectomy liver failure. 